\title{
Collaboration among Physicians and Nurses in Intensive Care Units: A Qualitative Study
}

\author{
Yasemin Akbal Ergun ${ }^{1}$ \\ Marmara University \\ Aysegul Yildirim Kaptanoglu ${ }^{3}$ \\ Trakya University
}

\author{
Fevzi Akinci ${ }^{2}$ \\ King's College \\ Jessica Wagner ${ }^{4}$ \\ King's College
}

\begin{abstract}
A health care transition project is currently underway in Turkey with the purpose of improving health care access, quality, and continuity of care by increasing patients' and health care professionals' satisfaction and cost effectiveness. Consequently, inter-professional collaboration in providing health care has a crucial role in increasing healthcare access, quality, and continuity of care in Turkey. This study aims to determine the factors influencing the collaborative experiences of nurses and physicians at intensive care units (ICUs). A qualitative research approach is used to determine how Turkish ICU nurses and physicians collaborate. Focus group, narrative analysis, and phenomenological approach methods are used to determine a qualitative framework for collaborations between nurses and physicians. A purposive sample of twelve physicians and twelve nurses were selected, and data were analyzed utilizing the content analysis method. The study was conducted in nine ICUs of three hospitals affiliated with two universities in Istanbul. Collaboration with three sub-themes (high, medium, and low) was identified. The results reveal an insufficient level of collaboration between physicians and nurses. Collaboration was identified as insufficient except for those nurses and physicians who had previously known each other. This relationship remains complex and unresolved, and needs to be understood and managed by ICU team leaders.
\end{abstract}

\section{Keywords}

Collaboration • Physicians • Nurses • Intensive care • Gender • Qualitative study

1 Department of Nursing, Marmara University, Maltepe, Istanbul Turkey. Email: yergun@marmara.edu.tr

2 The William G. McGowan School of Business, King’s College, Wilkes-Barre, PA, USA. Email: fevziakinci@kings.edu

3 Correspondence to: Aysegul Yildirim Kaptanoglu (PhD), Department of Health Management, Faculty of Health Sciences, Trakya University, Balkan Campus, Edirne 22030 Turkey. Email: jessica.wagner@siemens.com

4 The William G. McGowan School of Business, King’s College, Wilkes-Barre, PA, USA. Email: jlb410@psu.edu \& phjess@yahoo.com

Citation: Akbal Ergun, Y., Akinci, F., Yildirim Kaptanoglu, A., \& Wagner, J. (2017). Collaboration among physicians and nurses in intensive care units: A qualitative study. Sanitas Magisterium, 3, 25-35. http://dx.doi.org/10.12738/SM.2017.1.0030 
Collaboration is defined as collective action towards obtaining a common goal in the spirit of trust and harmony (D'Amour, Goulet, Pineault, Labadie, \& Remondin, 2004). However, in the context of health care management, collaboration is understood as the interaction between physician and nurse in making clinical decisions and problem solving (Baggs, Ryan, Phelps, Richeson, \& Johnson 1992; Baggs et al., 1999). The essential condition of collaboration is direct and open communication with respect for various perspectives and blended with mutual responsibility for problem solving.

In the past few decades, this collaboration between nurses and physicians has been extensively investigated (Fredheim, Danbolt, Havett, Kjonsberg, \& Lien, 2011; Schmalenberg \& Kramer, 2009). Yildirim et al. (2005; p. 436) state, "Attitudes toward collaboration vary significantly by type of institution, job classification, physician's gender, and physician's employment status." Hojat et al. (2003; p. 432) state, "Nurses desire collaborative nurse-physician relationships more than physicians, regardless of cultural background and independent of gender and age."

Hospitals are multi-professional healthcare settings in which inter-professional collaboration between physicians and nurses is important for patient care (Hojat et al., 2003). In the absence of collaboration, patients' outcomes (particularly in the clinical context of patient care) are negatively influenced. In addition, collaboration breaks down when nurses feel that their views and findings are not considered.

Nurses perceive their success depends on having the ability to share their ideas and opinions. Nurses perceive themselves as successful to the extent that they share their ideas and opinions with team members (Ardahan, Akcasu, \& Engin, 2010). When there is close collaboration between physicians and nurses in the ICU, and when nurses have high decision-making autonomy, the result is better patient outcomes (Papathanassoglou, Tseroni, Karydaki, Vazaiou, Kassikou, \& Lavdaniti, 2005).

The main purpose of this study is to investigate the nurse/physician collaborations in the ICU. Our hypothesis is that collaboration among nurses and physicians is high because ICU physician and nursing roles are somewhat equalized in order to obtain mutual benefit in treating patients with very severe conditions. Although ICUs are ranked among the highest collaborative places for health professionals (i.e., physicians, nurses, and other skilled health professionals) in the hospital, the collaborative relationship between them is inadequate (Hansson, Arvemo, Marklund, Gedda, \& Mattsson, 2010; Yildirim, Aktaş, \& Akdaş, 2006).

In fact, while nurses benefit from this collaboration by gaining social status, physicians utilize nurses as keen and clever observers in the monitoring process of critically ill patients. 


\section{Purpose}

This study aims to determine the factors influencing the collaborative experiences of nurses and physicians at ICUs.

\section{Method}

\section{Research Design}

The qualitative method has been chosen for this study as this method has the ability to produce rich content from the collaborative challenges experienced by nurses and physicians. Focus group methodology through group interviews were utilized (Silverman, 2010). Utilizing focus groups endeavors to create a natural communicative context on collaboration relationships in the ICU where health professionals can share experiences by primarily talking to one another without a researcher present. Focus groups were employed for drawing out physicians' and nurses' opinions and experiences. The aim was to more deeply search and understand the feelings, attitudes and opinions towards collaboration.

\section{Universe and Sampling}

The study was conducted at Marmara University and Istanbul University's affiliated hospital ICUs. The study used university-affiliated hospitals because they have fully functioning ICUs. Istanbul University has two affiliated hospitals with five ICUs: a Neonatal ICU, Coronary Care ICU, Trauma ICU, Medical ICU, and Respiratory ICU. Marmara University has one affiliated hospital with four ICUs: a Neonatal ICU, Coronary ICU, Trauma ICU, and Medical ICU.

The focus group participants were strategically selected by the researchers to represent all of the intensive care physicians and nurses in the hospitals. The study sample was chosen to reflect variations in age, gender, professional experiences, and specialties in ICUs. Thirty-six health professionals participated in the study, 18 physicians and 18 nurses.

The authors selected three focus groups from each affiliated hospital, and participants engaged in a total of three discussions. The moderator's primary role is to introduce the three main questions adopted by Hojat et al.'s (2003) nurse-physician collaboration scale, which had been translated into Turkish by Yildırım et al., 2006.

We examined collaboration levels (high, medium, low) by asking the following three main questions:

i. What are the strengths and weaknesses of collaboration between physician and nurse in the ICU with respect to your experiences? 
ii. What skills and qualities do you believe are essential for providing collaboration with each other in order to care for patients?

iii. How do you think this collaboration is influenced by gender?

Another role of the moderator was to ensure that each member of the focus group was aware of every question and that each member of the group was focused on the topic of the study. The moderator participated in the focus groups by asking additional questions and observing interactions between nurses and physicians. Brief notes during the focus group were taken by one of the authors as a reporter. All interviews were audiotaped and then transcribed.

\section{Data Analysis}

Analysis of the study was conducted using the systematic text condensation process through narrative analysis. Brief summaries and encoded notes taken by the reporter were classified, some of which the researchers extracted.

In other words, all transcriptions of each focus group session were reviewed and coded line-by-line to allow for data analysis (Yildirim \& Şimşek, 2008). To describe focus groups' statements on collaboration, researchers conducted initial coding in categories of units of meaning. After this was completed, phenomenological methods were used as a complementary method to each narrative. These methods focused on nurses' and physicians' experiences with respect to introspection and empathetic thinking. All interviews were 35-60 minutes in length, recorded with the participants' permission, and transcribed verbatim. Notes were taken concurrently.

The Ethics Committee of Istanbul University approved the protocol of this study. The administrations of the participating hospitals also formally approved the study's methodology.

\section{Results}

The findings reflect key issues from the analysis through three levels of collaboration:

1. Total collaboration (high-level) means sharing power and organizational settings for patients' needs.

2. Partial collaboration (medium-level) is defined as professional interaction generally supported by nurses for difficult clinical circumstances.

3. Low-level collaboration is the use of ICU protocols and pathways. 
There are two other thematic clusters: interpersonal knowledge/communication/ professional recognition and mutual accessibility to medical and nursing services.

\section{Levels of Collaboration}

Nurses and physicians were asked what their strengths and weaknesses are in collaborations between physicians and nurses in the ICU with respect to their experiences. The participants aimed to identify what the term collaboration meant, while the interviewers aimed to identify what the term collaboration meant to the participants.

In this study, twelve nurses (coded A, B, C, D, E, F, G, H, I, J, K, L) and twelve physicians (coded Z, Y, X, W, V, U, T, S, R, Q, P, O) described their collaboration level.

Twelve physicians (seven female, five male physicians [V, U, T, S, R]) participated. All physicians had at least two years of ICU experience. Six were specialized as a surgeon, intern, pediatrician, or anesthesiologist. Six were in the process of specializing in surgery, internal medicine, pediatrics, or anesthesiology.

Twelve nurses (three male [C, F, H], nine female) were included in the study. Their nursing experience ranged from 2 to over 10 years.

The nurses and physicians stated having an average of 5.4 years $(S D=1.6)$ of employment history with each other in the same ICU. These same nurses and physicians expressed that the quality of health services were influenced positively by high collaborative practices in the ICU.

\section{Collaboration and Interpersonal Knowledge}

(A, female) said, "I have been working in this hospital and ICU for 6 years; every nurse and physician knows me. They also rely on the fact that I do my work properly, carefully, and meticulously." Communication and cooperation tend to be effective when nurses and physicians are familiar with each other. Two consulting physicians (V, 45, male; O, 50, female) said, "It is easier to have good and high collaboration levels when the staff knows each other. While recognizing and sharing the same ICU, nurses and physicians experience mutual and beneficial collaboration."

For the question, "What skills and qualities do you believe are essential for collaborating with each other in order to care for patients?", Nurses C (27, male) and $\mathrm{F}(25$, male) said, "The physician is not serious about there being a professional situation in the ICU. We who know each other seem to collaborate well, but the physician does not know our name and only gives orders calling us 'sister.' Even though we know their names, we call them 'sir." 
Nurses L (22, female) and E (25, female) agreed with their colleagues' statement. "Even in the ICU we don't have the opportunity to be professional or collaborative."

\section{Mutual Accessibility and the Case of Gender in the Work Place}

For the question, "How do you think this collaboration can be influenced by gender?." nurses did not feel accepted by physicians as independent professionals. In Turkey, some nurses complete vocational nursing high school and can begin to work in the healthcare system. Most nurses therefore do not have university degrees and are under the control of physicians as an auxiliary health professional (Yildirim et al., 2005).

I do not have balanced work between my heavy duties and responsibilities. Even though I notice a patient problem, I do not have the right to declare my opinions. When I inform physicians, they focus on the physical part of the body without using a holistic approach. I am closer with the patients than the physicians are. But when I talk with physicians about my opinions on patient treatment they tell me to look after my own work. (Nurse D, 26, female; Nurse H, 29, male)

Dr. U (29, male), Dr. S (30, male), and Dr. R (26, male) said, "We are training residents. Nurses are with the patients for a longer time than us in the ICU. So if a nurse explains something reasonable to us, we accept it. But nurses' body language is more important to us."

Nurses' manners are very important in the collaboration process. If they want to act friendly, they can make me aware of this and must do it for the patient's sake, but a nurse must not discus my orders in front of the patient or the head consulting physician. (Dr. T, resident, male, 30)

Dr. P (consultant; 53, female) said, "Health care professionals in the ICU seems to be lazy. Nurses are used to accepting our orders but they do not want to accept young and inexperienced physicians."

Dr. X (resident, 33, female) said, "I try to be friendly towards the nurses but they interpret my friendliness incorrectly."

Dr. W (resident, 33, male) said, "Because of the patriarchal structure of society, women tend to obey men. This is wrong socialization! However, conflicts can also be seen within the same gender in professional life."

Nurses always state that they do not like auxiliary health personal terminology. They think of themselves as the physicians' servants. When the physician is female, they get frustrated by her commands. There is conflict between them. Everybody's doing their job. Physicians feel independent, but nurses do not. (Dr. Q, resident, 34, male) 
It is easier working with male physicians than female physicians. Female physicians think they are superior because they have more education than a nurse. Female physicians are mostly rude to female nurse, but female physicians try to be gentler with male nurses. (Nurse I, female; 24)

Nurse J (female, 27) said, "Sometimes it's also the same for male physicians, but mostly they are more polite."

Nurse K (female, 31) said, "I donไt think so, because the behaviors of physicians depend on character type and personality."

Nurse L (female, 30) said, "I do believe that if physicians and nurses are careful at work and write down their orders, the collaboration process will be more feasible. Collaboration between nurses and physicians speeds up the process of job professionalization."

\section{Discussion}

The findings of this study reveal three different type of collaboration: high, medium, and low. There are inequalities in education in terms of gender in Turkey. In bigger families, women are less likely to attend school. Female students and workers are more demotivated but are ambitious. Therefore, female workers such as nurses and physicians need time to develop more professional relations in the work environment.

Gender is one of the many aspects of cultural diversity in Turkey. As health professionals, one must be able to do what is best for the patient (from both the patient's and doctor's perspectives) regardless of one's personal characteristics. The history of male-dominated medical practice also affects this situation.

However, two main factors facilitate establishing collaboration between nurses and physicians. First, the length of time the nurse and physician have worked together in the same ICU; physicians and nurses build a relationship of mutual respect the longer they work together in the same hospital or ICU. Mutually understanding professional roles might improve collaborative practices between physician and nurse. Our findings reveal nurses and physicians want to improve the collaboration process in the ICU but problems about inter-professional relations exist between them.

Collaborative communication can be associated with positive patient, nurse, and physician outcomes. However, intervention-focused research that seeks to improve collaborative communication has been lacking up to now. The pretest-posttest repeated-measures design incorporated baseline data collection and implemented the intervention over 8 months followed by two data collections, immediately afterwards followed by one 6 months later. In the end, ICU nurse and physician leaders' 
communication skills were determined to have improved significantly. Leaders also reported increased satisfaction with their own communication and leadership skills. In addition, nursing- and physician-staff perceptions of nursing leadership and problem-solving between groups increased. Staff nurses reported lower personal stress, even though they perceived significantly more situational stress. This study finding provides evidence that collaborative communication between nurses and physicians can be improved.

Miller (2001) examined the collaborative interactions between nurses and physicians in ICUs and saw they are significantly related to mortality rates and length of stay in the units. For this reason, collaborative interaction should be an integral part of quality improvement programs. ICUs can use this example to incorporate an assessment of collaborative interaction levels into their quality improvement program.

One article in Critical Care Medicine investigated the association of collaboration between ICU physicians and nurses with patient outcome (Baggs et al., 1999). The study examined patients that were ready to transfer out of the ICU and provided questionnaires that were used to assess care providers' reports of collaboration in making the transfer decision. After checking for severity of illness, the association between inter-professional collaboration and patient outcome was assessed, and unitlevel organizational collaboration and patient outcomes were ranked. The results of Baggs et al.'s study determined Medical ICU nurse reports of collaboration were associated positively with patient outcomes. No other association between individual reports of collaboration and patient outcome were found. There was a perfect rank order correlation between unit-level organizational collaboration and patient outcome across the three units. It concluded that the study offered some support for the importance of collaboration between physicians and nurses in ICU care delivery, a variable susceptible to intervention and further study.

One article securing collegial/collaborative nurse-physician (CCRNMD) relationships mentioned in its first section that the goal of this multisite, evidencebased, management practice initiative was to identify structures that enable CCRNMD relationships (Schmalenberg, et al., 2005). In it, the authors discussed the methodology and selection of the sample of 141 physicians, managers, and staff nurses from 44 clinical units in five hospitals that had previously demonstrated extensive CCRNMD relationships. These 141 experts were interviewed to identify structures enabling CCRNMD relationships. The first section presents the structures that enable CCRNMD relationships as described and tested in the literature, as well as a description of the characteristics of the clinical units, experts, and CCRNMD relationships found in this study. In its second section, the structures identified by experts as needed for securing CCRNMD relationships is presented, along with suggestions for achieving them. 
Boyle and Kochinda (2004) examined and tested an intervention to enhance collaborative communication among nurse and physician leaders; the communication skills of ICU nurse and physician leaders were determined to have improved significantly. Nurse and physician clinical meetings help to professionalize collaboration in the ICU. These meetings help to reduce misunderstandings and disagreements between them.

In previous studies (Nayeri \& Negarandeh, 2009; Sapountzi-Krepia, Psychogiou, Sakellari, Kostandinidou, \& Dimitriadou, 2007), researchers reported that ICU patients cared for by nurses and physicians who worked collaboratively had lower "acuity-adjusted" mortality rates than did patients cared for by less collaborative nurses and physicians. Collaboration in ICU results with fewer deaths and reflects back onto the ICU. Physicians have formal relationships but don't have collaborative ones (Knaus, Draper, Wagner, \& Zimmerman, 1986; Larson, 1999). Working in the same hospital and same ICU is very important for good collaborative practices.

The objective of their article, Communication between Physicians and Nurses as a Target for Improving End-of-Life Care in Intensive Care Units: Challenges and Opportunities for Moving Forward (Puntillo \& McAdam, 2006), was to discuss obstacles and barriers to effective communication and collaboration regarding end-of-life issues between nurses and physicians in ICUs. To evaluate practical interventions for improving communication and collaboration, we undertook a systematic literature review. An increase in shared decision making can result from a better understanding and respect for the perspectives and burdens felt by other caregivers. ICU nurses value their contributions to end-of-life decision making and want to have a more active role. Increased collaboration and communication can result in more appropriate care and increased physician, nurse, patient, and family satisfaction. Recommendations for improving communications between ICU physicians and nurses include joint rounds, patient-care seminars, and interprofessional dialogues. Communication interventions such as using daily-rounds forms, communication training, and a collaborative practice model have shown positive results. When communication is clear and constructive, and practice is truly collaborative, the end-of-life care provided to ICU patients and families by satisfied and engaged professionals will improve markedly.

\section{Conclusion}

Research has proven that collaboration between nurses and physicians needs to have shared responsibility and professionalism in patient-care processes. Understanding one another's position will aid collaboration between these two main professions of the health care team. Nurses and physicians are faced with medical decisions that 
must be made instantly under difficult circumstances. Collaborative training and education is necessary for nursing and medical students (e.g., Introduction to Clinical Practice program). This training is a good opportunity for students to learn positive collaborative practices. Without collaboration, an outcome of good healthcare for patients and practice in ICUs is less likely.

Nurses' and physicians' characteristics play an important role in collaboration, and behaviors play a significant role in collaboration-practice management.

\section{References}

Ardahan, M., Akcasu, B., \& Engin, E. (2010). Professional collaboration in students of medicine faculty and school of nursing. Nurse Education Today, 30(4), 350-354.

Baggs, J. G., Ryan, S. A., Phelps, C. E., Richeson, J. F., \& Johnson, J. E. (1992). The association between interdisciplinary collaboration and patient outcomes in a medical intensive care unit. Heart Lung, 21(1), 18-24.

Baggs, J. G., Schmitt M. H., Mushlin, A. I., Mitchell, P. H., Eldredge, D. H., Oakes, D., \& Hutson, A. D. (1999). Association between nurse-physician collaboration and patient outcomes in three intensive care units. Critical Care Medicine, 27(9), 1991-1998.

Boyle, D. K, \& Kochinda, C. (2004). Enhancing collaborative communication of nurse and physician leadership in two intensive care units. Journal of Nursing Administration, 34, 60-70.

D’Amour, D., Goulet, L., Pineault, R., Labadie, J. F., \& Remondin, M. (2004). Comparative study of inter-organizational collaboration and its effects in four Quebec socio-sanitary regions: The case of perinatal care. Universite de Montreal. Retrieved from http:/www.ferasi.umontreal.ca/ fra/07_info/Rapport\%20ANG.pdf

Fredheim, T., Danbolt, L. J., Havett, O. R., Kjonsberg, K., \& Lien, L. (2011). Collaboration between general practitioners and mental health care professionals: A qualitative study. International Journal of Mental Health Systems, 5(1), 13. http://dx.doi.org/10.1186/1752-4458-5-13

Hansson, A., Arvemo, T., Marklund, B., Gedda, B., \& Mattsson, B. (2010). Working together Primary care doctors' and nurses' attitudes to collaboration. Scandinavian Journal of Public Health, 38(1), 78-85.

Hojat, M., Gonnella, J. S., Nasca., T. J., Fields, S. K., Cicchetti, A., Lo Scalzo, A., ... Torres-Ruiz A. (2003). Comparisons of American, Israeli, Italian and Mexican physicians and nurses on the total and factor scores of the Jefferson scale of attitudes toward physician-nurse collaborative relationships. International Journal of Nursing Studies, 40(4), 427-435.

Knaus, W. A., Draper, E. A., Wagner, D. P., \& Zimmerman, J. E. (1986). An evaluation of outcome from intensive care in major medical centers. Annals of Internal Medicine, 104(3), 410-418.

Larson, E. (1999). The impact of physician-nurse interaction on patient care. Holistic Nurse Practitioner, 3(2), 38-46.

Miller, P. A. (2001). Nurse-physician collaboration in an intensive care unit. American Journal of Critical Care, 10(5), 341-350.

Nayeri, N. D., \& Negarandeh, R. (2009). Conflict among Iranian hospital nurses: A qualitative study. Human Resource Health, 7, 25. http://dx.doi.org/10.1186/1478-4491-7-25 
Papathanassoglou, E. D. E., Tseroni , M., Karydaki, A., Vazaiou, G., Kassikou, J., \& Lavdaniti, M. (2005). Practice and clinical decision-making autonomy among Hellenic critical care nurses. Journal of Nursing Management, 13(2), 154-164.

Puntillo, K., \& McAdam, J. L. (2006). Communication between physicians and nurses as a target for improving end-of-life care in the intensive care unit: Challenges and opportunities for moving forward. Critical Care Medicine, 34(11), 332-340.

Sapountzi-Krepia, D., Psychogiou, M., Sakellari, E., Kostandinidou, A., \& Dimitriadou, A. (2007). How Greek nurses and nursing students define nursing: A qualitative content analysis. Health Science Journal, 1(3). Retrieved from http://www.hsj.gr/volume1/issue3/qualitative.pdf

Schmalenberg, C., \& Kramer, M. (2009). Nurse-physician relationships in hospitals: 20,000 nurses tell their story. Critical Care Nurse, 29, 74-83.

Schmalenberg, C., Kramer, M., King, C., Krugman, M., Lund, C., Poduska, D., \& Rapp, D. (2005). Excellence through evidence: Securing collegial/collaborative nurse-physician relationships, Part 1. Journal of Nursing Administration, 35(10), 450-458.

Silverman, D. (2010). Interpreting qualitative data. London, UK: Sage Publication.

Yildirim, A., \& Şimşek, H. (2008). Sosyal bilimlerde nitel araştırma yöntemleri [Qualitiative research methods in the social sciences] ( $7^{\text {th }}$ ed.). Ankara, Turkey: Seçkin Yayıncılik.

Yildirim, A., Akinci, F., Ates, M., Ross, T., Issever, H., Isci, E., \& Selimen, D. (2006). Turkish version of the Jefferson scale of attitudes toward physician-nurse collaboration: A preliminary study. Contemporary Nurse, 23(1), 38-45.

Yildirim, A., Aktaş, T., \& Akdaş, A. (2006). Collaboration among medical and nursing school student. TAF Preventative Medicine Bulletin, 5(3), 166-175.

Yildirim, A., Ates, M., Akinci, F., Ross, T., Selimen, D., Issever, H., ... Akgün, M. (2005). Physiciannurse attitudes toward collaboration in Istanbul's public hospitals. International Joural of Nurse Studies, 42(4), 429-437. 
